\title{
Aplicação da escala SF-36 em pacientes operados de tumores da base do crânio
}

\author{
Katiane R. Servelhere ${ }^{1}$, Yvens B. Fernandes ${ }^{2 *}$, Ricardo Ramina ${ }^{3}$, Guilherme Borges ${ }^{4}$
}

\section{RESUMO}

Introdução: A base do crânio é uma região que pode ser afetada por diferentes tipos de tumores. Os tumores dessa região podem ser benignos ou malignos e se localizarem na cavidade anterior, média ou posterior do crânio. Embora relativamente raras, essas lesões podem afetar significativamente os pacientes em virtude de sua localização anatômica complexa e riscos próprios do manejo cirúrgico. A qualidade de vida de pacientes operados de tumores da base do crânio pode ser prejudicada não somente pelo comprometimento neurológico resultante da lesão anatômica, mas também por problemas psicossociais tais como diminuição da autoconfiança e autoestima, mudança nas atividades de vida diária, dependência, estigma, discriminação, dificuldade de interação social, desemprego, entre outras. Dessa forma, o objetivo deste estudo foi avaliar a qualidade de vida dos pacientes operados de tumores da base do crânio. Método: O estudo incluiu 38 indivíduos que foram avaliados entre seis meses e um ano após a cirurgia usando a escala de qualidade de vida SF-36. Resultados: Todos os pacientes tiveram pontuação acima de 50. Em seis dos oito domínios, nos componentes físico e mental, mulheres pontuaram melhor que homens, especialmente em LAF (limitação por aspectos físicos). Quando comparadas as idades, pacientes acima de 50 anos pontuaram melhor. Quando comparados os sexos, mulheres até 50 anos pontuaram melhor que os homens. Por outro lado, homens com idade superior a 50 anos pontuaram melhor que as mulheres. Conclusão: Embora haja diferenças, estas não foram estatisticamente significantes. Assim, não houve melhora significativa nos quesitos social, físico, psicológico e funcional quando idade e sexo foram comparados.

\section{PALAVRAS-CHAVE}

Base de crânio, SF-36, tumor, qualidade de vida.

\begin{abstract}
Application of SF-36 scale in patients operated on skull base tumors

Background: The skull base is a region that may be affected by different types tumors. Tumors in this region can be benign or malignant and are located in the anterior fossa, middle or posterior fossa. Although relatively rare, they are potentially harmful, because of the complex anatomic features of these regions and the inherent risk of a surgical procedure. The quality of life of patients can be hampered not only by neurological impairment, but also by psychosocial problems such as decreased self-confidence and self-esteem, change in daily activities, dependency, stigma and discrimination, difficulty in social interaction and unemployment. Thus, the aim of this study was to assess the quality of life of patients operated on for tumors of the skull base. Method: The study included 38 individuals who were evaluated between six months and one year after surgery, using the SF-36. Results: All patients had scores above 50 when all domains were summed up. In six out of eight domains (physical and mental components) women scored better than men, especially in LAF (limitation due to physical abilities). When compared to age, patients older than 50 years scored better. When compared with gender, women until 50 years had a better score. On the other hand, men aged over 50 years scored better than women. Conclusion: Although there are slight differences, these were not statistically significant. We concluded that no significant improvement occurred in social, physical, psychological and functional outcome when age and sex were compared.
\end{abstract}

\section{KEYWORDS}

Skull base, SF-36, tumor, quality of life.

1. Fisioterapeuta, Departamento de Neurologia, Faculdade de Ciências Médicas da Universidade Estadual de Campinas (Unicamp), Campinas, SP, Brasil.

2. Ph.D., professor-associado, Departamento de Neurologia, Faculdade de Ciências Médicas da Unicamp, Campinas, SP, Brasil.

3. MD, Ph.D., Departamento de Neurocirurgia do Instituto Neurológico de Curitiba, Brasil, pós-graduado em cirurgia pela Pontifícia Universidade Católica (PUC) de Curitiba, Brasil.

4. Ph.D., Departamento de Neurologia, Faculdade de Ciências Médicas da Unicamp, Campinas, SP, Brasil. 


\section{Introdução}

A importância de temas enfocando qualidade de vida $(\mathrm{QV})$ em pacientes com tumores está sendo cada vez mais estudada. A QV é definida como um conceito multidimensional que considera ao menos quatro dimensões: física, social, psicológica e funcional. ${ }^{1} \mathrm{~A} \mathrm{QV}$ vem sendo usada para avaliar modalidades de tratamento e promover a restauração das atividades de vida diária e o retorno dos pacientes às suas atividades sociais e de trabalho. ${ }^{2}$

Recentemente, QV e qualidade de vida relacionada à saúde (QVRS) estão sendo reconhecidas por profissionais da saúde para avaliar o resultado dos tratamentos. ${ }^{1}$ A percepção dos pacientes sobre os efeitos da doença na vida diária, estado de saúde e sua QV é reconhecida como sendo importante para mensurar o resultado do procedimento cirúrgico. ${ }^{4.5}$ Embora esses pacientes sejam usualmente uma boa fonte de informação sobre suas satisfações e necessidades, tais informações sobre a QV durante o período de doença ou tratamento não vêm sendo rotineiramente coletadas em clínicas de pesquisa ou na prática médica. ${ }^{3,6}$

Segundo Gil et al. (2004), a compreensão detalhada de diferentes aspectos da QV pode ajudar cirurgiões a melhorarem a avaliação e o tratamento desses pacientes, identificarem impedimentos específicos tão cedo quanto possível durante o período de seguimento e especificarem intervenções médicas para pacientes com alto risco de resultados negativos. ${ }^{7}$

As cirurgias de base do crânio são complexas, com risco inerente de déficits importantes e até mesmo de óbito no período pós-operatório. No entanto, muitas dessas lesões são benignas e a remoção total ou subtotal pode ser alcançada, permitindo o controle da doença, a estabilização ou mesmo a cura a longo prazo. Preservar a QV e a independência é o objetivo final. ${ }^{8}$

A QV em pacientes operados de tumores da base do crânio pode ser dificultada não somente por déficit neurológico resultante da lesão anatômica, mas também por vários problemas psicológicos como diminuição da autoconfiança e autoestima, modificação das atividades cotidianas, dependência, estigma e discriminação; dificuldade de interação social, desemprego, entre outros. Além disso, as atitudes e expectativas dos familiares podem influenciar negativamente na QV desses pacientes. ${ }^{9}$ Dentre os instrumentos genéricos para mensurar saúde, QV e estado subjetivo de bem-estar físico e social, a SF-36 é um dos mais amplamente usados, por causa de suas características de compreensibilidade, brevidade e altos padrões de confiabilidade e validez, sendo um dos menos extensos existentes até o momento. ${ }^{4,10,14}$

O objetivo deste estudo foi avaliar a QV em pacientes operados de tumor da base do crânio usando a escala de qualidade de vida SF-36. Especificamente, objetivamos identificar o grupo de pacientes (homens ou mulheres) que obtiveram a maior pontuação nas oito categorias da SF-36, componentes físico e mental de acordo com sexo e idade, e também verificar se esses indivíduos se encontravam em um grau satisfatório de independência física, social psicológica e funcional.

\section{Material e método}

Este é um estudo transversal com análise pós-cirúrgica em 38 indivíduos diagnosticados com tumores benignos da base do crânio, durante o período de 1998 a 2003.

A fim de selecionar e convidar os pacientes e/ou responsáveis para participar desta pesquisa, todas as informações sobre os objetivos e procedimentos do estudo foram relatados. A concordância com o estudo foi confirmada mediante assinatura do termo de consentimento livre e esclarecido.

O projeto foi aprovado pelo Comitê de Ética e Pesquisa da Faculdade de Ciências Médicas da Unicamp (516/2006).

O estudo incluiu pacientes submetidos a procedimento cirúrgico de tumor da base do crânio, e a análise dos resultados foi obtida por meio de uma entrevista por paciente usando o questionário SF-36. O SF-36 (The Medical Outcomes Study 36 - Item Short-Form Health Survey) é adequado para autoadministração, administração por meio do computador ou por um indivíduo com idade superior a 14 anos e treinado, por meio de telefone. ${ }^{3} \mathrm{O}$ SF-36 é uma escala de saúde curta que abrange somente 36 questões, ${ }^{12}$ divididas em oito domínios. Os oito domínios representam aqueles mais frequentemente mensurados na avaliação da saúde. $\mathrm{Na}$ versão traduzida e culturalmente adaptada para o Brasil, esses domínios são designados como: Capacidade Funcional (CF), Aspectos Sociais (AS), Aspectos Emocionais (AE), Aspectos Físicos (AF), Vitalidade (VIT), Saúde Mental (SM), Dor e Estado Geral de Saúde (EGS). Cada categoria varia de 2 a 10 itens e todas elas podem ser resumidas em dois componentes: Físico e Mental.

O componente físico inclui os domínios CF, AF, Dor e EGS, e o componente mental inclui SM, AE, AS e VIT, embora os domínios EGS e VIT também possam estar diretamente correlacionados com o outro componente. Essa divisão destina-se a visualizar, de forma mais ampla, os dois principais componentes que podem estar envolvidos de forma diferente na avaliação de QV dos pacientes que se submeteram a extirpação de tumor de base de crânio. O domínio CF avalia limitações devidas ao estado de saúde, que vão desde o banho e vestir-se até atividades vigorosas. O domínio AF avalia as limitações na forma e 
na quantidade de trabalho e como essas limitações dificultam a realização dos trabalhos e atividades da vida diária dos pacientes. O domínio Dor tem uma questão sobre a intensidade e outra cujo objetivo é medir sua extensão ou interferência nas atividades diárias do paciente. O domínio EGS reflete a percepção do paciente acerca de sua saúde, variando de excelente a muito ruim, se ele se considera bem ou doente. Os cinco itens de avaliação de SM incluem uma ou mais questões sobre as quatro dimensões-chave da saúde mental que são: ansiedade, depressão, alterações de comportamento ou descontrole emocional e bem-estar psicológico. O domínio $\mathrm{AE}$ avalia dificuldades na capacidade de trabalhar ou realizar outras atividades diárias devido a problemas emocionais. O domínio AS objetiva analisar a integração dos indivíduos na vida social e também avalia se a participação em tais atividades é afetada por problemas de saúde. O domínio VIT é composto por quatro itens que consideram tanto o nível de energia quanto o nível de fadiga. Finamente, há uma questão comparativa entre o estado de saúde geral e a percepção que o paciente tem dele em comparação a um ano atrás. ${ }^{13}$

A escala avalia aspectos negativos da saúde (doença) e aspectos positivos (bem-estar). O SF-36 é capaz de discriminar vários estágios e a gravidade da doença e não se limita à retrospecção por longos períodos de tempo, porque se centra apenas em um período de quatro semanas. ${ }^{14}$

Os 38 indivíduos foram avaliados com o SF-36 por profissionais da saúde durante um período de seis meses a um ano após suas cirurgias. Cada avaliação foi realizada em salas destinadas a consultas médicas no Hospital das Clínicas da Universidade Estadual de Campinas (Unicamp). A todos os indivíduos selecionados foi aplicada a escala de qualidade de vida Medical Outcomes Study 36 - Item Short-Form Health Survey (SF-36) traduzida e validada para o português.

Os dados registrados no roteiro para avaliação da escala SF-36 e suas respectivas pontuações foram revisados manualmente pela pesquisadora responsável e depois foram transcritos na forma de arquivos para o banco de dados do programa computacional Excel 2003, como indicado pela Comissão de Pesquisa e Estatística da Faculdade de Ciências Médicas da Unicamp. Os dados do programa computacional Excel foram repassados para o programa de análise estatística SAS, no qual foi feita a análise descritiva dos dados. A análise estatística foi realizada mediante consultoria e suporte oferecidos pela Comissão de Pesquisa e Estatística da Unicamp.

Os dados foram analisados descritivamente por frequências absolutas (n) e relativas (\%). Para as variáveis categóricas e contínuas, foram usados a média, o desvio-padrão, a mediana, o primeiro e o terceiro quartil e os valores máximos e mínimos. Para comparar os escores de QV segundo o sexo e a faixa etária, inicialmente foi utilizado o teste de Shapiro-Wilk com objetivo de verificar se havia aderência dos escores à curva de distribuição normal. Os escores que apresentaram aderência à curva de distribuição normal foram comparados aplicando-se o teste t-Student, e nos escores que não apresentaram aderência à curva de distribuição normal foi utilizado o teste de Mann-Whitney. O software SAS versão 9.1.3 (SAS Institute Inc., Cary, NC, USA, 2002-2003) foi utilizado na análise dos dados e o nível de significância estatística de 5\% foi adotado.

\section{Resultados}

Como o estudo não apresentou um grupo controle, os pacientes foram comparados entre si. A comparação foi feita com relação a sexo, idade (acima e abaixo de 50 anos) e classificação entre aqueles que estavam acima ou abaixo da nota de corte de 50 pontos. A tabela 1 mostra que a faixa etária variou de 20-74 anos, com média e desvio-padrão de $49,7(12,1)$. Verificou-se que a amostra foi constituída predominantemente por mulheres $(65,8 \%)$.

A tabela 2 mostra a distribuição dos escores para cada categoria do SF-36. A maior média e o desvio-padrão foi de 72,9 (22,1) para EGS, seguido por CF com média e desvio-padrão de $71,3(24,4)$. A menor média e desvio-padrão foi de 58,3 (40,3) para o domínio AF. Para todos os domínios, os escores foram acima de 50.

A comparação dos escores de acordo com o gênero é mostrada na tabela 3. Para seis dos oito domínios (AF, EGS, V, AS, AE e SM), bem como para os componentes físico e mental, mulheres pontuaram melhor que os homens, e para os domínios CF e DOR, os homens pontuaram melhor do que as mulheres. Embora haja diferenças nos escores entre homens e mulheres, elas não foram estatisticamente significantes.

A tabela 4 compara a pontuação total dos pacientes por faixa etária. Para a maioria dos domínios, os pacientes com 50 anos de idade ou mais jovens apresentaram melhor desempenho do que os pacientes com mais de 50 anos. Contudo, somente em um domínio essa diferença tendeu à significância (AF).

Tabela 1 - Análise descritiva da idade e sexo dos pacientes

\begin{tabular}{lccc} 
Média & \multicolumn{2}{c}{49,7} & \\
Desvio-padrão & & 12,1 & \\
Mediana & & 51,0 & \\
Mínimo & & 20,0 & \\
Máximo & & 74,0 & \\
Sexo & n & & $\%$ \\
Mulheres & 25 & & 65,8 \\
Homens & 13 & & 34,2 \\
\hline
\end{tabular}

n: número de pessoas. 


\begin{tabular}{|c|c|c|c|c|c|}
\hline Domínios e componentes & $\mathbf{M}$ & DP & Mediana & Mín & Máx \\
\hline Capacidade funcional (CF) & 71,3 & 24,4 & 75,0 & 20 & 100 \\
\hline Aspectos físicos (AF) & 58,3 & 40,3 & 58,3 & 0 & 100 \\
\hline Dor & 68,3 & 28,2 & 63,0 & 10 & 100 \\
\hline Estado geral de saúde (EGS) & 72,9 & 22,1 & 77,0 & 25 & 100 \\
\hline Vitalidade (VIT) & 61,8 & 21,0 & 60,0 & 25 & 100 \\
\hline Aspectos sociais (AS) & 67,4 & 24,8 & 62,5 & 25 & 100 \\
\hline Aspectos emocionais (AE) & 65,3 & 34,3 & 66,6 & 0 & 100 \\
\hline Saúde mental (SM) & 64,3 & 23,3 & 72 & 20 & 100 \\
\hline Componente físico & 67,7 & 22,8 & 73,3 & 20,5 & 100 \\
\hline Componente mental & 65,4 & 19,8 & 66,2 & 23,8 & 100 \\
\hline
\end{tabular}

M: média; DP: desvio-padrão; Min: mínimo; Máx: máximo.

\begin{tabular}{|c|c|c|c|c|c|}
\hline \multirow[t]{2}{*}{ Escores } & \multicolumn{2}{|c|}{ Mulheres } & \multicolumn{2}{|c|}{ Homens } & \multirow[t]{2}{*}{ p valor } \\
\hline & $\mathbf{M}(\mathrm{DP})$ & (Mín;Máx) & $\mathrm{M}(\mathrm{DP})$ & (Mín;Máx) & \\
\hline PCF & $70,4(21,8)$ & $(20-100)$ & $73,1(29,5)$ & $(20-100)$ & $0,4534^{\mathrm{a}}$ \\
\hline RAF & $66,7(40)$ & $(0-100)$ & $42,3(37,3)$ & $(0-100)$ & $0,0736^{a}$ \\
\hline BDor & $67(26,9)$ & $(22-100)$ & $70,9(31,5)$ & $(10-100)$ & $0,6849^{\mathrm{a}}$ \\
\hline GEGS & $73,4(20,3)$ & $(35-100)$ & $72(26,1)$ & $(25-100)$ & $0,8543^{\mathrm{a}}$ \\
\hline VVIT & $65,1(21,4)$ & $(25-100)$ & $55,4(19,4)$ & $(25-100)$ & $0,1808^{\mathrm{a}}$ \\
\hline AAS & $69,4(25,6)$ & $(25-100)$ & $63,5(23,6)$ & $(37,5-100)$ & $0,4480^{\mathrm{a}}$ \\
\hline RAE & $66(33,5)$ & $(0-100)$ & $64,1(37,2)$ & $(0-100)$ & $0,9489^{\mathrm{a}}$ \\
\hline MSM & $64,6(26,2)$ & $(20-100)$ & $63,7(17,6)$ & $(28-88)$ & $0,9074^{\mathrm{b}}$ \\
\hline PCfísico & $69,3(20,1)$ & $(26,8-97,5)$ & $64,6(27,7)$ & $(20,5-100)$ & $0,5488^{b}$ \\
\hline MCMental & $67,4(20,9)$ & $(23,8-100)$ & $61,7(17,7)$ & $(31-97)$ & $0,4072^{b}$ \\
\hline
\end{tabular}

M(DP): média (desvio-padrão); Mín: mínimo; Máx: máximo.

${ }^{a}$ Test Mann-Whitney; ${ }^{\text {bTestet-Student. }}$

Tabela 4 - Comparação geral da pontuação do SF-36 de acordo com a idade

\begin{tabular}{|c|c|c|c|c|c|c|c|}
\hline & \multicolumn{3}{|c|}{$\leq 50$ anos } & \multicolumn{3}{|c|}{$>50$ anos } & \multirow[t]{2}{*}{ P valor ${ }^{*}$} \\
\hline & $\mathbf{n}$ & M & DP & $\mathbf{n}$ & M & DP & \\
\hline CF & 18 & 66,9 & 27,6 & 20 & 75,3 & 21 & 0,4535 \\
\hline $\mathrm{AF}$ & 18 & 44,4 & 39,8 & 20 & 70,8 & 37,4 & 0,0736 \\
\hline DOR & 18 & 64,3 & 32,1 & 20 & 72 & 24,5 & 0,6849 \\
\hline EGS & 18 & 69,6 & 26 & 20 & 76 & 18 & 0,8543 \\
\hline VIT & 18 & 59,7 & 19,6 & 20 & 63,6 & 22,6 & 0,1875 \\
\hline AS & 18 & 62,5 & 23,1 & 20 & 71,8 & 26 & 0,448 \\
\hline $\mathrm{AE}$ & 18 & 57,4 & 33,9 & 20 & 72,5 & 33,9 & 0,9489 \\
\hline SM & 18 & 66,2 & 23,3 & 20 & 62,6 & 23,9 & 0,8181 \\
\hline Cfísico & 18 & 61,3 & 26,7 & 20 & 73,5 & 17,3 & 0,6361 \\
\hline Cmental & 18 & 61,2 & 20,3 & 20 & 69,2 & 19,2 & 0,3164 \\
\hline
\end{tabular}

n: número de pessoas; M: média; DP: desvio-padrão; Cfísico: componente físico; Cmental: componente mental.

*Teste de Mann-Whitney.

\section{Discussão}

Ciconelli et al. avaliaram tradução, adaptação cultural e propriedades de medida (confiabilidade e validez) em pacientes com artrite reumatoide e concluíram que a versão brasileira da SF-36 foi um parâmetro reprodutivo e válido para uso na avaliação da QV de pacientes brasileiros com essa doença. Contudo, em neurologia, 
especificamente em cirurgia de tumor de base de crânio, não foram encontrados estudos brasileiros em que essa escala foi aplicada.

Este é o primeiro estudo brasileiro que mensura QV em pacientes submetidos à cirurgia de tumor de base de crânio usando a escala SF-36 como instrumento de avaliação de resultados pós-operatórios.

Alonso et al. avaliaram o impacto de condições crônicas comuns na QVRS entre a população geral em oito países (Dinamarca, França, Alemanha, Itália, Japão, Holanda, Noruega e Estados Unidos) por e-mail e entrevista transversal. A amostra variou de 2.031-4.084. A SF-36 foi usada em indivíduos com e sem condições crônicas. Mais da metade da amostra $(55,1 \%)$ relatou ao menos uma condição crônica e 30,2\% relataram mais de uma condição crônica. Hipertensão, alergias e artrite foram as condições crônicas mais frequentemente relatadas. Das doenças estudadas, a artrite teve o maior impacto na QVRS da população geral nos países estudados. ${ }^{15}$

Mosconi et al. utilizaram uma amostra de 165 pacientes com câncer de laringe objetivando produzir e validar o SF-36. Foi possível testar suas características em termos de aceitação dos pacientes, testes psicométricos e validade clínica. Globalmente, os resultados mostraram que, em todos os pacientes em diferentes fases da doença, o SF-36 teve desempenho muito bom. Todos os pacientes completaram o questionário. Os coeficientes de confiabilidade interna efetivamente repetiram os resultados satisfatórios relatados para a versão original do SF-36. Com relação à capacidade da escala em discriminar um determinado conceito de saúde em relação a variáveis clínicas, os resultados também foram bons. O estudo mostrou que o SF-36 foi bem aceito pelos pacientes e foi capaz de detectar o impacto de diferentes abordagens de tratamento na QVRS. ${ }^{1}$

Uma vez que a pontuação do SF-36 varia de 0-100, foi estipulado um valor de corte de 50 para determinar os pacientes que estão melhor e os que estão pior, devido à falta de um grupo controle. Os pacientes que pontuaram com menos que 50 estariam com a QV pior e aqueles que pontuaram acima de 50 estariam com boa QV. Assim, observou-se que, para o grupo total, a pontuação foi acima de 50, ou seja, os pacientes relataram boa QV.

\section{Correlação do SF-36 com idade}

Gil et al. ${ }^{7}$ desenvolveram e validaram um instrumento multidimensional de avaliação de QV específico para pacientes que se submeteram à cirurgia para tumores da base anterior do crânio. Para isso, foram revisados cinco questionários de QV, sendo um deles o SF-36. A principal razão de avaliar a $\mathrm{QV}$ nesses pacientes foi promover a restauração da função diária e acelerar seu retorno à vida cotidiana normal. A escala contém seis domínios: Desempenho, Função Física, Vitalidade, Dor, Influência nas emoções e Sintomas Específicos. Este estudo demonstrou que pacientes com idade acima de 60 anos tiveram pior pontuação que pacientes mais jovens para o domínio Desempenho (ou seja, desempenho geral e no trabalho, desempenho em casa e como a saúde afeta esse desempenho, participação em atividades sociais e comunicação com as pessoas) e Função Física (inclinar o tronco para a frente e ficar, caminhar, subir escadas e conduzir as atividades de vida diária). Dessa forma, a idade teve impacto negativo na QVRS em atividades leves como tomar banho e se vestir, moderadas como caminhar e usar aspirador de pó e vigorosas como correr e levantar objetos pesados. Além disso, houve prejuízo na realização de alguns trabalhos ou atividade diária regular em consequência da piora da saúde física.

Tufarelli et al. ${ }^{14}$ também observaram que seus pacientes com idade acima de 45 anos pontuaram significativamente pior em cinco domínios após cirurgia para retirada de neurinoma do acústico. Betchen et al. ${ }^{15}$ corroboraram os achados de Tufarelli et al. e Gil et al., já que seus pacientes com idade até 55 anos pontuaram significativamente melhor do que os pacientes com idade superior a 55 anos para os domínios EGS e CF. Opostamente, Nicoucar et al. ${ }^{16}$ verificaram que o grupo até 55 anos de idade ou mais jovem tendeu a pontuar pior do que o grupo acima de 55 anos em todos os domínios do SF-36. O estudo atual está de acordo com os achados de Nicoucar et al., pois nossos pacientes com idade acima de 50 anos também tenderam a pontuar melhor que os pacientes até 50 anos ou mais jovens em quase todos os domínios, especialmente em LAF $(p=0,07)$.

$O$ presente estudo mostrou que pacientes com idade superior a 50 anos tenderam a pontuar melhor que os pacientes mais jovens. Contudo, a maioria dos estudos na literatura mostrou o oposto a isso. De acordo com Nicoucar et al. ${ }^{16}$, pacientes mais jovens podem não ter tido graves problemas médicos anteriormente (provavelmente não passaram por cirurgia de grande porte no passado), enquanto os pacientes mais velhos podem já ter experimentado mais problemas médicos e, portanto, foram capazes de lidar melhor com uma nova perda de capacidade. Desse modo, explica-se o motivo de pacientes mais velhos pontuarem melhor do que os mais novos.

\section{Correlação do SF-36 com o sexo}

Tufarelli et al. observaram que as mulheres estavam piores que os homens em todos os domínios do SF-36 $(\mathrm{p}<0,05)$ após cirurgia de neurinoma do acústico. Para 
Betchen et al., as mulheres também pontuaram menos do que os homens em todos os domínios, no entanto somente em $\mathrm{CF}$ e AF essas pontuações foram relevantes. Igualmente, Nicoucar et al. encontraram que mulheres com shwannoma vestibular pontuaram notavelmente abaixo em habilidades físicas quando comparadas aos homens com o mesmo tipo de tumor. Betchen et al. reconhecem que isso ocorre quando comparados os sexos na população normal; dessa forma, há tendência de as mulheres pontuarem menos, e isso não se limita à cirurgia de neurinoma do acústico.

\section{Correlação do SF-36 com sexo e idade}

Ao contrário dos estudos de Tufarelli et al., Betchen et al. e Nicoucar et al., no presente estudo mulheres com idade até 50 anos pontuaram melhor que homens da mesma idade na maioria dos domínios, principalmente em AF. Este estudo também mostrou que homens com idade acima de 50 anos pontuaram melhor que as mulheres da mesma idade. Alguns estudos mostraram a influência da idade no estado de saúde dos pacientes, ${ }^{7,16,17}$ mas neste estudo não foi possível provar a interferência da idade na QV dos pacientes, provavelmente por falta de um grupo controle. $\mathrm{O}$ estudo atual revelou que, quando gênero e idade são comparados com os domínios da SF-36, não houve diferença estatisticamente significante acima ou abaixo de 50 anos, apesar de os homens mais velhos pontuarem melhor que as mulheres da mesma idade.

\section{Comparação dos estudos que utilizaram o tratamento cirúrgico para pacientes com tumores da base do crânio e o grupo controle por meio da SF-36}

Kelleher et al. ${ }^{17}$ avaliaram a QVRS em pacientes diagnosticados com tumores da base do crânio usando o SF-36 e compararam os escores com os da população normal. Esse estudo mostrou piora significativa em todos os domínios do SF-36 para o grupo de tumor, exceto no domínio Dor. Martin et al. ${ }^{18}$, quando avaliaram seus pacientes operados de neurinoma do acústico e os compararam com um grupo controle, encontraram notável diminuição em todos os domínios do SF-36 para o grupo operado, exceto Dor, SM e LAE.
Tufarelli et al. também compararam a QV de seus pacientes após cirurgia de neurinoma do acústico com a QV da população normal e verificaram que a pontuação do SF-36 para os pacientes foi inferior em todos os domínios. Da Cruz et al. ${ }^{20}$, quando fizeram a comparação de pacientes tratados cirurgicamente de neurinoma do acústico com a população saudável por meio do SF-36, constataram que somente o domínio VIT foi melhor para os pacientes operados. Cheng et al. ${ }^{12}$ encontraram essa diminuição nos escores do SF-36 para os pacientes operados de shwannoma vestibular, porém só foi significativa para AF. Segundo os autores desse estudo, essa diminuição pode estar relacionada com disfunção do nervo facial, disfunção vestibular, zumbido ou perda auditiva que pode persistir após a cirurgia. Seis pacientes do atual estudo apresentaram hipoacusia e seis apresentaram anacusia, porém não foi possível correlacionar a maioria desses sintomas com um tipo específico de tumor e a QV dos pacientes em virtude de a amostra ser pequena.

Rameh e Magnan ${ }^{19}$ compararam a QV de pacientes após a cirurgia de shwannoma vestibular via translabiríntica (TLA) e retrosigmoide (RSA) com grupo controle por meio do SF-36. Ambos os grupos de pacientes tiveram escores significativamente menores no questionário, portanto qualidade de vida menos satisfatória. Porém, quando comparados os grupos TLA e RSA entre si, não houve relevância estatística em nenhum dos oito domínios. Rameh e Magnan acreditam que a deterioração da $\mathrm{QV}$ é provavelmente um efeito combinado da cirurgia e do processo da doença em si. O estudo atual também comparou os pacientes entre si e nenhum dos domínios revelou diferenças estatisticamente significantes.

Em conclusão, no presente estudo, a escala SF-36 aplicada em pacientes com tumor da base do crânio não mostrou melhora significativa na vida social, física, psicológica e funcional quando os grupos foram comparados. Novos estudos com recrutamento de um número maior de pacientes e grupo controle é necessário.

\section{Referências}

1. Mosconi P, Scid B, Cifani S, Statd, Crispino S, Fossati R, et al. The performance of SF-36 health survey in patients with larygeal cancer. Head Neck. 2000;22(2):175-82.

2. Gil Z, Abergel A, Spektor S, Cohen JT, Khafif A, Shabatai $E$, et al. Quality of life following surgery for anterior skull base tumors. Arch Otolaryngol Head Neck Surg. 2003;129(12):1303-9.

3. Ware JE, Gandek B. The SF-36 Health Survey: development and use in Mental Health Research and the IQOLA Project. Int J Ment Health. 1994;23(2):49-73. 
4. Lang DA, Neil-Dwyer G, Garfield J. Outcome after complex neurosurgery: the caregiver's burden is forgotten. J Neurosurgery. 1999;91:359-63.

5. Ciconelli RM. O SF-36 e o desenvolvimento de novas medidas de avaliação de qualidade de vida. Acta Reunatol Port. 2008;33:127-33.

6. Ware JE, Sherbourne CD. The MOS SF-36 Short-Form Health Survey (SF-36). Med Care. 1992;30(6):473-83.

7. Gil Z, Abergel A, Spektor S, Shabatai E, Khafif A, Fliss DM. Development of a cancer-specific anterior skull base quality-of-life questionnaire. J Neurosurg. 2004;100:813-9.

8. Westphal AC, Alonso NB, Silva TI, Azevedo AM, Caboclo LO, Garzon E, et al. Comparação da qualidade de vida e sobrecarga dos cuidadores de pacientes com epilepsia por esclerose mesial temporal e epilepsia mioclônica juvenil. J Epilepsy Clin Neurophysiol. 2005;11(Suppl 2):71-6.

9. Ciconelli RM. Tradução para o português e validação do questionário genérico de avaliação de qualidade de vida "Medical Outcomes Study 36-item Short-Form Health Survey (SF-36)" [PhD Thesis]. São Paulo: Escola Paulista de Medicina; 1997.

10. Ciconelli RM, Ferraz MB, Santos W, Meinão I, Quaresma MR. Tradução para a língua portuguesa e validação do questionário genérico de avaliação de qualidade de vida SF-36 (Br SF-36). Rev Bras Reumatol. 1999;39(Suppl 3):143-50.

11. Souza WA. Avaliação da adesão ao tratamento e dos resultados clínicos e humanísticos na investigação da hipertensão arterial resistente [PhD Thesis]. Campinas, SP: Universidade Estadual de Campinas; 2008.

12. Cheng S, Naidoo Y, Da Cruz M, Dexter M. Quality of life in postoperative vestibular schwannoma patients. Laryngoscope. 2009;119:2252-7.
13. Alonso J, Ferrer M, Gandek B, Ware JE, Aaronson NK, Mosconi $P$, et al. Health-related quality of life associated with chronic conditions in eight countries: results from the International Quality of Life Assessment (IQOLA) Project. Qual Life Res. 2004;13:283-98.

14. Tufarelli D, Meli A, Alesii A, Angelis E, Badaracco C, Falcioni $M$, et. al. Quality of life after acoustic neuroma surgery. Otology Neurototoly. 2006;27:403-9.

15. Betchen SA, Walsh J, Kalmon D. Self-assessed quality of life after acoustic neuroma surgery. J Neurosurg. 2003;99:818-23.

16. Nicoucar K, Momjian S, Vader JP, Tribolet N. Surgery for large vestibular schwannomas: how patients and surgeons perceive quality of life. 2006;105:205-12.

17. Kelleher MO, Fernandes MF, Sim DW, O'Sullivan MG. Health-related quality of life in patients with skull base tumors. Br J Neurosurg. 2002;16(Suppl 1):16-20.

18. Martin HC, Sethi J, Lang D, Neil-Dwyer G, Lutman ME, Yardley L. Patient-assessed outcomes after excision of acoustic neuroma: postoperative symptoms and quality of life. J Neurosurg. 2001;94:211-6.

19. Rameh C, Magnan J. Quality of life of patients following stages III-IV vestibular schwannoma surgery using the retrosigmoid and translabyrinthine approaches. Auris Nasus Larynx. 2010;37(Suppl 5):546-52.

20. Da Cruz JM, Moffat DA, Hardy DG. Postoperative quality of life in vestibular schwannoma patients measured by the SF36 Health Questionnaire. Laryngoscope. 2000;110:151-5.

Endereço para correspondência

Katiane Raisa Servelhere

Rua 13 de maio, 461, Taboão

12916-020 - Bragança Paulista, SP

Telefone: (11) 4033-7398

E-mail: kaservel@hotmail.com 